\title{
D-Dimer; A Potential Clinical Marker for Predicting Metastasis \& Stages in Lung Cancer
}

\author{
Faiz Ahmed Faiz' , Muhammad Tayyab ${ }^{2}$, Khalid Aftab ${ }^{3 *}$, Muhammad Aslam ${ }^{4}$ and Muhammad Fahad Faiz ${ }^{1}$ \\ ${ }^{1}$ Department of Pathology, Islam Medical College, Sialkot, Pakistan
}

${ }^{2}$ Department of Pathology, Sialkot Medical College, Sialkot, Pakistan

${ }^{3}$ Department of Pharmacology \& Therapeutics, Sialkot Medical College, Sialkot, Pakistan

${ }^{4}$ Department of Pathology, MIMC Mirpur, Pakistan

*Corresponding author: Khalid Aftab, Department of Pharmacology \& Therapeutics, Sialkot Medical College, Pakistan

\begin{abstract}
The relationship between plasma D-dimer level and the prognosis of advanced lung cancer is close to each other. This study investigated the role of plasma D-dimer as a prognostic factor in advanced lung cancer: a) The aim of the current study was to investigate the association of D dimer plasma level with the development of stages in lung cancer. b) D-dimers levels as predictor of fibrinolysis and Disseminated Intravascular Coagulation (DIC). The subjects were selected from oncology department, Ghulab Devi Hospital and Mayo Hospital, Lahore. Total 45 subjects were included in the present study. The selected subjects were divided into two groups. Group A included 15 normal, healthy, age and sex matched controls and group B included 30 patients, histologically diagnosed cases of lung cancer. The patients had no history of coagulation disorders or on anti-coagulant therapy. The plasma D-dimer was measured in normal, heathy Controls and 30 histologically diagnosed cases of lung cancer by enzyme-linked immunosorbent assay. The median age of the patients (18 males and 12 females ) was $39.433 \pm 5.11$ and $13.3 \%$ had stage lb, $20 \%$ had llb, $6.7 \%$ llla, $36.11 \mathrm{lllb}, 23.3 \%$ had IV disease. Histologic sub-type was Non-small cell lung carcinoma (NSCLC) $30 \%$, Small cell lung carcinoma (SCLC) were $10 \%$, Squamous cell carcinoma were $10 \%$, adenocarcinoma $16.7 \%$ and large cells carcinoma were $10 \%$.In the present study. The Mean \pm SD value of D-dimer level of the patients was $1068.70 \pm 441.86 \mathrm{ng} / \mathrm{dl}$, which was significantly higher than that of the control group. The plasma levels of D-dimer were significantly higher in patients with bronchogenic carcinoma as compared with healthy controls. Plasma D-dimer level was significantly higher in metastatic disease $(\mathrm{P}<0.01)$. D dimer levels were positively associated with clinical cancer stage $(\mathrm{P}<0.05)$ and metastasis $(\mathrm{P}<0.05)$. These findings suggested that the plasma $\mathrm{D}$ dimer level may be use as marker for predicting cancer metastasis and staging in lung cancer.
\end{abstract}

Keywords: Lung Cancer; Malignancies; Haemostatic System; Coagulation; Tumor Cells; Hyper coagulopathy; D Dimer

\section{Introduction}

Lung cancer is one of the most prevalent malignancies in the world $[1,2]$. This cancer is currently the most common malignant disease and the leading cause of cancer related-deaths in all age groups and in both sexes [3]. The association between cancer and haemostatic system has been known since Trousseau's study from the $19^{\text {th }}$ century [4]. Coagulation or fibrinolytic system activation is present in lung cancer patients at clinical or subclinical level. There is a complex interaction, which has an important role in the course of the disease, between pathogenetic mechanisms of thrombosis, tumor cells, homeostatic systems, and patient characteristics.
Patients with deep venous thrombosis (DVT) or subclinical hypercoagulopathy usually have worse prognosis. D-dimer is the cleavage product of cross linked fibrin that is formed by activation of the coagulation system, which signals hyperfibrinolysis in response to clot activation and fibrin formation [5]. Elevated levels of D-dimer have been detected in patients exhibiting diffuse intravascular coagulation [6], thromboembolic events, [7] D-dimer is a widely used biomarker for indicating the activation of coagulation and fibrinolysis [8,9]. Coagulation disorders are among the most common complications in cancer patients $[8,10]$. D-dimer is a degradation product of cross-linked fibrin that 
appears in the blood after a blood clot is degraded by fibrinolysis [11]. D-dimer is produced by fibrin degradation, and measurement of D-dimer levels can help in the diagnosis of thrombosis [12]. D-dimer levels have been found to be significantly higher in lung cancer with poor prognosis[13,14]. D-dimer is a plasmin-mediated degradation product of cross-linked fibrin clot, which is formed by the activation of the coagulation system for any reason [15]. Several studies suggested that serum D-dimer level may be an important parameter in assessing prognosis of disease and response to treatment in patients with lung cancer[14,16]. As D-dimer is a sensitive marker of fibrinolysis so, it is recommended measuring the plasma level of D-dimer in all new lung carcinoma patients [17]. The results suggested that the plasma level of D-dimer was notably associated with the extent of tumor metastasis and tumor stage in lung cancer patients.

\section{Material and Methods}

Total 45 subjects were included in the present study. 30 patients histologically diagnosed cases of bronchogenic carcinoma from oncology department, Ghulab Devi Hospital and Mayo Hospital Lahore, who were admitted between July 2017 to August 2018. The control group consisted of 15 healthy individual without co-morbidity. Lung cancer staging was performed for all patients according to the $7^{\text {th }}$ TNM classification. The inclusion criteria were as following histological diagnosed cases of bronchogenic carcinoma pretreatment were selected. Patients with a history of venous thrombosis or anticoagulation therapy, hypertension, cardiovascular and cerebrovascular disease, diabetes, acute or chronic inflammatory disease, or previous malignancy were excluded from the current study. Written informed consent was obtained from each subjects before the sample collection. $3 \mathrm{ml}$ of venous blood was collected in the disposable syringe and put in to vacutainer tubes containing anticoagulant citrate buffer (1:9, buffer blood for D-dimers assay. History and clinical features were recorded in all subjects on performa. The principals of different tests and their procedures were adopted accordingly. Blood samples were centrifuged and the plasma was separated. D-dimer level was measured in the plasma, via enzyme-linked fluorescent immunoassay method using a PC-Vidas device (BioMerieux Fr). A D-dimer level $<500 \mathrm{ng} / \mathrm{dl}$ was considered normal.

\section{Results and Observations}

Thirty patients with histological confirmed lung cancer were enrolled in the study. Mean age was $39.433 \pm 5.11$ years within the, range of $26-52$ years, and there were 18 males $(60.0 \%)$ and 12 females (40.0\%). There were 05 (16.7\%) adenocarcinomas, 10 (33.3\%) squamous cell carcinomas, 03 (10.0\%) small cell carcinomas and 09 (30\%) non small cell carcinomas. 04 (13.3\%) patients were classified as stage Ib, 06 (20\%) patients as stage IIb, $02(6.7 \%)$ patients as stage IIIa, $11(36.7 \%)$ patients as stage IIIb and $07(23.3 \%)$ patients as stage IV. The general characteristics of the lung cancer patients are shown in Table 1.
Table 1: Characteristics of the patients $(n=30)$

\begin{tabular}{|c|c|}
\hline Characteristic & Median (quartile) or frequency \\
\hline Gender (male/female) & $18 / 12$ \\
\hline Age (years) & $39.433 \pm 5.11$ \\
\hline Smoking (yes/no) & $22 / 08$ \\
\hline Histological type & \\
\hline Non-small cell lung carcinoma & 3 \\
\hline small cell lung carcinoma & 10 \\
\hline Squamous cells carcinoma & 5 \\
\hline Asenocarcinoma & 3 \\
\hline Large cell carcinoma & \\
\hline Stages of lung cancer & 4 \\
\hline Stage lb & 6 \\
\hline Stage llb & 2 \\
\hline Stage lla & 11 \\
\hline Stage lllb & 7 \\
\hline Stage iv & \\
\hline
\end{tabular}

D-dimer levels in patients with lung cancer were higher than those of the control group, and the difference was statistically significant P 0.05 (Table 2). D-dimer levels according to disease stage are shown in Table 3. When the D-dimer levels were compared according to disease stage in patients with lung cancer, a significant difference was observed between the D-dimer levels of patients with stage $\mathrm{lb}$, llb and those with stage IV disease ( $\mathrm{P}=0.025)$. There was no significant difference between the other stage groups in terms of D-dimer levels. D-dimer levels were significantly higher in lung cancer patients than in the control group $(\mathrm{P}<0.01$; Table 3$)$. These data suggested that the plasma level of $\mathrm{D}$ dimer may be used as a marker of metastasis and staging in lung cancer.

Table 2: D-dimer in patients of lung cancer and control group.

\begin{tabular}{|c|c|c|}
\hline & Lung cancer & Control group \\
\hline & patients $(\mathrm{n}=30)$ & $(\mathrm{n}=15)$ \\
\hline & Mean $\pm S D$ & Mean $\pm S D$ \\
\hline D-dimer $(\mathrm{ng} / \mathrm{dl})$ & $1068.70 \pm 441.86$ & $308.8 \pm 111.580$ \\
\hline
\end{tabular}

Table 3: D-dimer levels according to stages in lung cancer (ng/ $\mathrm{dl})$.

\begin{tabular}{|c|c|c|c|}
\hline \multicolumn{4}{|c|}{ D-dimer } \\
\hline Stages & Mean \pm SD & Min-max & P \\
\hline IB & $634 \pm 59.44$ & $550-695$ & $\mathrm{P}=0.025$ \\
\hline IIB & $940 \pm 284.09$ & $695-870$ & $\mathrm{P}>0.05$ \\
\hline IIIA & $905 \pm 100.62$ & $710-1010$ & $\mathrm{P}>0.05$ \\
\hline IIIB & $\begin{array}{c}1129.22 \pm \\
221.86\end{array}$ & $700-1478$ & $\mathrm{P}>0.05$ \\
\hline IV & $1728.60 \pm$ & $695-2200$ & $\mathrm{P}=0.025$ \\
\hline
\end{tabular}

\section{Discussion}

There is some evidence that the activation of coagulation and fibrinolytic system by neoplastic cells facilitates invasiveness 
and metastases [18]. Thus, the extent of such activation has been associated with tumor stage and prognosis in some malignancies such as breast, colorectal and lung cancer $[19,20]$. D-dimer is a product of cross-linked fibrin degradation by plasmin-induced fibrinolytic activity, and D-dimer levels are a biomarker of global hemostasis and fibrinolysis [21]. High D-dimer levels were associated with poor prognoses in patients with breast, [22,23] colon and rectum, $[24,19]$ and lung $[25,26]$ cancers. In the present study, it was demonstrated that elevated plasma levels of D dimer were associated with clinical cancer stages and metastasis in lung cancer patients. D-Dimers: In this study, D-dimers were found to be significantly increased $(p<0.01)$ in patients with lung cancer when compared with controls (Table 2). The D-dimer level was more in SCLC as compared to NSCLC. The increased D-dimer was more in Patients having DIC and in patients of metastatic disease. These increased levels of D-dimers may be due to enhanced fibrinolysis [27] in their study Showed increased levels of D-dimers in patients having malignancy. These findings are consistent with the results of $[27,18,28,29]$. Several studies have reported that plasma D-dimer levels were elevated and associated with the stage and mortality in lung cancer $[28,29]$. They also observed similar increase of D-dimers in lung cancer (2.0). Our study has some limitations. This is a retrospective observational study with small sample size. Therefore, the findings of this study need to be validated by prospective and multicentre studies. The present study thus provides more reference values for patients with later-stage tumors and poorer overall condition. SCLC is a highly invasive tumor with a poor prognosis, with different biological characteristics from NSCLC. Valid biomarkers are therefore needed to determine the prognosis of SCLC. In the present study, pretreatment plasma D-dimer levels were independently in patients with SCLC. This conclusion is supported by previous studies of lung cancer in general.

\section{Conclusion}

The present study demonstrated that D-dimer plasma level was significantly higher in lung cancer patients and associated with clinical stages and metastasis. The current study was limited in that it only identified the association of D-dimer with tumor stage and metastasis.

\section{References}

1. Wahab M, Boroumand N, Castro C, El Zeky F, Eltorky M (2007) Changing trends in the distribution of the histologic types of lung cancer, a review of 4, 439 cases. Ann Diagn Pathol 11(2): 89-96.

2. Tas D, Okutan O, Kaya H, Kartaloglu Z, Kunter E (2008) Analysis of 138 cases of lung cancer in a training hospital compared to the data of lung cancer cases diagnosed ten years previously. Marmara Med J 21(3): 231237.

3. Pujari VV, Lokhande RM, Meshra SH, Waghmare RD (2016) Clinical and pathological presentations of bronchogenic carcinoma in a tertiary care centre. J Evolution Med Dent Sci 5 (47): 3068-3071.

4. Falanga A, Rickles FR (1999) Pathophysiology of the thrombophilic state in cancer patient. Semin Tromb Hemost 25(2): 173-182.
5. Jiang X, Mei X, Wu H, Chen X (2017) D dimer level is related to the prognosis of patients. Ann Transl Med 5 (20): 394.

6. Kobayashi T, Kajiki M, Nihashi K and Honda G (2017) Surveillance of the safety and efficacy of recombinant human soluble thrombomodulin in patients with obstetrical disseminated intravascular coagulation. Thromb Res 159: 109-115.

7. Kwietniak M, Al Amawi T, Błaszkowski T, Sulżyc Bielicka V, Kładny J (2017) The usefulness of D dimer in diagnosis and prediction of venous thromboembolism in patients with abdominal malignancy. Pol Przegl Chir 89 (3): 27-30.

8. Horowitz NA, Blevins EA, Miller WM, Perry AR, Talmage KE, et al. (2011) Thrombomodulin is a determinant of metastasis through a mechanism linked to the thrombin binding domain but not the lectin-like domain. Blood 118(10): 2889-2895.

9. Muschel RJ (2004) Coagulation facilitates tumor cell spreading in the pulmonary vasculature during early metastatic colony formation. Cancer Res 64(23): 8613-8619.

10. Huang W and Kim HR (2015) Dynamic regulation of platelet derived growth factor D (PDGF-D) activity and extracellular spatial distribution by matriptase-mediated proteolysis. J Biol Chem 290(14): 9162-9170.

11. Hu Q Wu Y, Tang J, Zheng W, Wang Q, et al. (2014) Expression of polycystins and fibrocystin on primary cilia of lung cells 1 . Biochem Cell Biol 92(6): 547-554.

12. Adam SS, Key NS and Greenberg CS (2009) D dimer antigen: current concepts and future prospects. Blood 113(13): 2878-2887.

13. Ma X, Li Y, Zhang J, Huang J, Liu L (2014) Prognostic role of D dimer in patients with lung cancer: a meta-analysis. Tumour Biol 35(3): 21032109.

14. Buccheri G Ferrigno D Ginardi C, Zuliani C (1997) Haemostatic abnormalities in lung cancer: prognostic implications. Eur J Cancer 33(1): 50-55.

15. Blomback B, Hessel B, Hogg D, Therkildsen L (1978) A two-step fibrinogen fibrin transition in blood coagulation. Nature 275(5680): 501-555.

16. Pavey Hawson SJ, GAT, Marsh NA (1999) Alterations to the fibrinolytic enzyme system in patients with non small cell lung carcinoma. Blood 10(5): 261-267.

17. Kobayashi T, Taguchi O, Gabazza EC, Yasui H, Yoshida M, et al. (1997) Prognostic significance of plasma D-dimer levels in patient with lung cancer. Thorax 52(6): 563-565.

18. Oya M, Akiyama Y, Okuyama T, Ishikawa H (2001) High preoperative plasma D-dimer level is associated with advanced tumor stage and short survival after curative resection in patients with colorectal cancer. Jpn J Clin Oncol 31(8): 388-394.

19. Blackwell K, Haroon Z, Broadwater G, Berry D, Harris L, et al. (2000) Plasma D-dimer levels in operable breast cancer patients correlate with clinical stage and axillary lymph node status. J Clin Oncol 18(3): 600608.

20. Fukumoto K, Taniguchi T, Usami N, Kawaguchi K, Fukui T, et al. (2015) Preoperative plasma D-dimer level is an independent prognostic factor in patients with completely resected non-small cell lung cancer. Surg Today 45(1): 63-67.

21. Batschauer AP, Figueiredo CP, Bueno EC, Ribeiro MA, Dusse LM, et al. (2010) D-dimer as a possible prognostic marker of operable hormone receptor negative breast cancer. Ann Oncol 21(6): 1267-1272.

22. Blackwell K, Hurwitz H, Lieberman G, Novotny W, Snyder S, et al. (2004) Circulating D-dimer levels are better predictors of overall survival and disease progression than carcinoembryonic antigen levels in patients with metastatic colorectal carcinoma. Cancer 101(1): 77-82.

23. Altiay G, Ciftci A, Demir M, Kocak Z, Sut N, et al. (2007) High plasma D-dimer level is associated with decreased survival in patients with lung cancer. Clin Oncol (R Coll Radiol) 19(7): 494-498. 
24. Chen Y, Yu H, Wu C, Li J, Jiao S, et al. (2016) Prognostic value of plasma D-dimer levels in patients with small-cell lung cancer. Biomed Pharmacother 81: 210-217.

25. Khan MZ, Tayyib Tassneem T, Farooq M, Ikram Din Ujjan, et al. (2004) A comparative study of FDPs and D-dimers in patients with breast cancer. Annals 10(4): 468-469.

26. Inal T, Anar C, Polat G, Ünsal I, Halilçolar H (2015) The prognostic of D-dimer in lung cancer. Clin Respir J 9(3): 305-313.

27. Komurcuoglu B, Ulusoy S, Gayaf M, Guler A, Ozden E (2011) Prognostic value of plasma D-dimer levels in lung carcinoma. Tumori 97(6): 743748.

(C) (P) This work is licensed under Creative

To Submit Your Article Click Here: Submit Article

DOI: $10.32474 /$ LOJPCR.2019.01.000108
28. Antoniou D, Pavlakou G, Stathopoulos GP, Karydis I, Chondrou E, et al. (2006) Predictive value of D-dimer plasma levels in response and progressive disease in patients with lung cancer. Lung Cancer 53(2): 205-210.

29. Kalemkerian GP, Loo BW, Akerley W, et al. (2017) NCCN Clinical Practice Guidelines in Oncology (NCCN Guidelines). Small Cell Lung Cancer Version 3. Fort Washington: National Comprehensive Cancer Network.

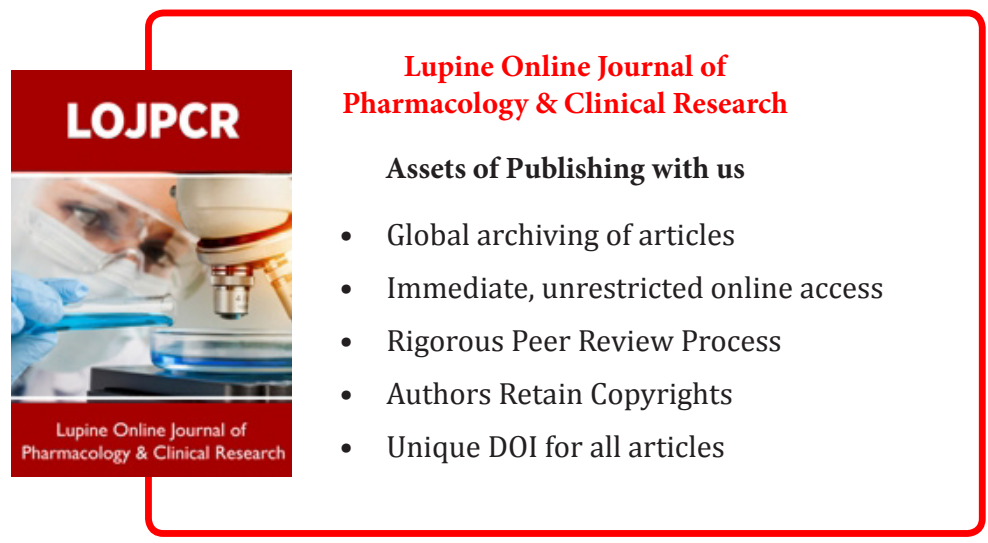

